\title{
Analysis of Platforms and Functions of Mobile-Based Personal Health Record Systems
}

\author{
Byung Kwan Choi ${ }^{1}$, Young-Taek Park ${ }^{2}$, Lee-Seung Kwon ${ }^{3}$, Yeon Sook Kim ${ }^{4}$ \\ ${ }^{1}$ School of Medicine, Pusan National University, Busan, Korea \\ ${ }^{2}$ Research Institute for Health Insurance Review and Assessment, Health Insurance Review \& Assessment Service, Wonju, Korea \\ ${ }^{3}$ Department of Health Care Management, Catholic Kwandong University, Gangneung, Korea \\ ${ }^{4}$ Department of Nursing, California State University, San Bernardino, CA, USA
}

Objectives: Little is known about the platforms and functionalities of mobile-based personal health record (PHR) applications. The objective of this study was to investigate these two features of PHR systems. Methods: The unit of analysis was general hospitals with more than 100 beds. This study was based on a PHR survey conducted from May 1 to June 30, 2020 and the National Health Insurance administrative data as of March 31, 2020. The study considered the platform, Android and iPhone operation system (iOS), and types of functionalities of PHR systems. Among the 316 target hospitals, 103 hospitals had adopted PHR systems. A logistic regression analysis was used. Results: This study found that 103 hospitals had adopted mobile-based PHR systems for their patients. Sixty-four hospitals (62.1\%) were adopting both Android and iOS, but $36(35.0 \%)$ and $3(2.9 \%)$ hospitals were adopting Android only or iOS only, respectively. The PHR systems of hospitals adopting both platforms were more likely to have functions for viewing prescriptions, clinical diagnostic test results, and upcoming appointment status compared to those adopting a single platform $(p<0.001)$. The number of beds (odds ratio [OR] = 1.004; confidence interval [CI], 1.001-1.007; $p=0.0029)$ and the number of computed tomography systems (CTs) per 100 beds (OR $=6.350$; CI, 1.006-40.084; $p=0.0493$ ) were significantly associated with the adoption of both platforms. Conclusions: More than $60 \%$ of hospitals had adopted both Android and iOS platforms for their patients in Korea. Hospitals adopting both platforms had additional functionalities and significant association with the number of beds and CTs.

Keywords: Personal Health Records, Electronic Medical Records, Electronic Health Records, Information Systems, Mobile Applications

Submitted: August 28, 2020

Revised: October 5, 2020

Accepted: October 23, 2020

\section{Corresponding Author}

Young-Taek Park

Health Insurance Review \& Assessment Service (HIRA), 60, Hyeoksin-ro, Wonju 26465, Korea. Tel: +82-33-739-0931, E-mail: pyt0601@hira.or.kr (https://orcid.org/0000-0002-7574-4165)

This is an Open Access article distributed under the terms of the Creative Commons Attribution Non-Commercial License (http://creativecommons.org/licenses/by$\mathrm{nc} / 4.0 /$ ) which permits unrestricted non-commercial use, distribution, and reproduction in any medium, provided the original work is properly cited.

(c) 2020 The Korean Society of Medical Informatics

\section{Introduction}

Many hospitals are providing various mobile applications to their patients whereby patients can view their health information and logs of hospital visits and can communicate with healthcare providers $[1,2]$. Digital data collected from individual patients while receiving healthcare and during their daily lives and stored by healthcare organizations (HOs) are called personal health records (PHRs). PHRs are electronic collections of information on individuals' health, healthcare, and well-being; the individual and authorized personnel can add, store, manage, and use such information in online, secure, and usable manners [3]. Computer systems, networks 
maintaining PHRs, and various applications are referred to as a PHR system.

There are many PHR systems, such as "MyChart" developed by Cleveland Clinics [4], "My Health at Vanderbilt" provided by Vanderbilt University Medical Center [5], and "My HealtheVet", a PHR portal run by the Department of Veterans Affairs in the United States [6]. The PHR applications of patients provide various functions, such as accessing records, scheduling appointments, setting reminders, and requesting referrals [7]. According to a recent study, more frequent portal use was observed after mobile access was added to PHR systems [8].

There have been several unsuccessful attempts to build PHRs by major technology companies. Google ran "Google Health" from 2008 to 2011 and Microsoft Corporation launched a PHR platform, "Health Vault", in 2007, but it came to an end on November 20, 2019 [9-11]. In contrast, Apple introduced Apple Health Record based on the iPhone operating system (iOS) with the 11.3 beta version in January 2018 [12]. By using mobile applications provided by Apple, patients can access their PHRs. By cooperating with many healthcare delivery organizations (HDOs), Apple enabled its application users to access their clinical information in HDOs. Many HDOs, including hospitals and clinics, have already participated in this network [13].

Generally speaking, patients' mobile PHR applications run on two types of platforms, Google's Android and Apple's iOS. For example, Iowa PHR running in Iowa State offers both iOS and Android platforms to users [14]. Apple Health Record is only based on an iOS platform. Regarding these two platforms, Android has greater openness for application development and data sharing, but less security [15] because many manufacturers and developers are participating in its application and hardware development. Thus, there are various Android models and wide variation in capacities among applications. In contrast, the iOS platform has contrasting features with less openness and high security. The iOS also has a good graphic user interface due to Apple's direct involvement in the development of the operating systems, iOS applications, and hardware.

In healthcare markets, it is frequently observed that some HOs provide two different PHR applications to their patients-one runs on Google's Android platform and the other runs on Apple's mobile platform. Although there have been many studies on information technology (IT) platforms $[16,17]$, little research has been conducted on PHR platforms at the organizational level.

This study predicted that hospitals with advanced techno- logical infrastructure, for example, those having advanced diagnostic medical equipment, are more likely to adopt both platforms rather than having a single platform. Patients who require expensive diagnostic tests could be considered important customers for those HOs having a large amount of medical equipment because the cost of taking an advanced diagnostic test is very high; thus, those customers could be main sources of revenue. Therefore, the possibility of running both platforms increases as the amount of such equipment increases.

The contingency theory proposes various ideas. Among these, one of the arguments may support our prediction. It states that effective organizations move in the direction fitting their structure with internal or external environments [18]. As previously mentioned, hospitals with the advantages of a having a large amount of medical equipment would adopt various PHR platforms to satisfy their customer needs. In contrast, hospitals that do not have those advantages would hesitate to invest in and to adopt various PHR platforms because their current IT infrastructure needs updating and requires more financial investment. The findings of some empirical studies indirectly support our prediction. Hospitals with a higher level of technological infrastructure were more likely to adopt the full Electronic Medical Record systems and vice versa for hospitals with lower levels [19]. Hospitals having better quality of care were more interested in adopting IT [20].

Many countries have keenly watched the progress of their PHR systems and those of other nations [21-23]. There are many barriers and facilitators associated with PHR adoption and use, and those barriers usually are related to technological, organizational, financial, and legal issues [24]. This study deals with these issues regarding the adoption of a PHR platform.

The objective of this study was to investigate the current adoption status of mobile PHR platforms by hospitals, their functions, and factors associated with PHR platform adoption. Factors identified through this study will provide various important results to policymakers, PHR vendor industries, and health information systems in foreign countries regarding how HOs behave with respect to the adoption of PHR platforms.

\section{Methods}

\section{Study Design and Data Sources}

A cross-sectional study design was adopted, and the unit of analysis was general hospitals. To achieve the proposed 
study objectives, a PHR survey of all general hospitals with 100 or more beds in Korea was conducted from May 1 to June 30, 2020. A professional researcher having a medical doctor degree managed the survey. The names of the general hospitals and their information, such as address, number of beds, and location were obtained from a publicly accessible website (https://opendata.hira.or.kr/home.do), "Healthcare Bigdata Hub" (HBH), provided by the Health Insurance Review and Assessment Service (HIRA). Regarding the process of the survey, market research on the PHR system developers and vendors was first conducted. Developers, vendors, their products, platforms, functionalities, and HOs installing those products were identified. Thus, it was easy to confirm which hospitals had installed PHR systems. One example was a software development company, Lemon Healthcare, which provides more than 40 PHR products to HOs. After the first step was completed, the remaining hospitals that were not identified in the first stage were checked to determine whether they had adopted PHR systems from the App Store, Google Play, or other business entities and search engines, such as Naver and Google. After the second stage, the survey data was merged with the health insurance administrative data from HIRA's HBH. This study was approved by the Institutional Review Board of the Pusan National University Hospital (No. H-2004-026-090) on April 28, 2020.

\section{Outcomes and Independent Variables}

The aim of the study is to investigate what types of PHR platforms HOs are adopting, the functionalities of PHRs, and factors associated with the type of platform adoption. This study descriptively defined a PHR platform as an operating system running a user's PHR applications and measured the adoption of PHR platforms with two categories. One was the adoption of both Android and iOS platforms, and the other was the adoption of a single platform, either Android or iOS. Regarding the functions of PHR platforms, this study focused on three availabilities: (1) scheduling appointments or viewing appointment status, (2) viewing past prescriptions or current prescription status, and (3) viewing lab test results or lab test scheduling.

Regarding the main independent variables, this study defined HOs' infrastructure as various advanced diagnostic equipment and measured this based on two items: the number of computed tomography systems (CTs) and magnetic resonance imaging machines (MRIs). Among various medical equipment, this study selected these two items because CTs and MRIs are relatively expensive, and they are critical sources of revenue for HOs. Therefore, HOs might be very interested in customers who may require CT and MRI imaging, which would affect the HO's PHR platforms.

For the other hospital covariates, the following variables were used: type of ownership (private vs. public), location (mega-metropolitan city or not), type of hospital (tertiary or not), and years of hospital operation. Mega-metropolitan locations were coded as administrative districts having more than 1,000,000 or more residents, and other locations were coded as having fewer residents than this number. All these data were from the $\mathrm{HBH}$ portal. The date used for measuring these variables was obtained on March 31, 2020.

\section{Statistical Analysis}

First, the descriptive statistics of independent variables were considered in terms of adoption types of PHR platforms. The functionalities of PHR systems were also investigated according to the types of platform adopted. The type of PHR platform was measured as either the adoption of both Android and iOS platforms or the adoption of a single platform, either Android or iOS. For the numeric and categorical measurement, $t$-tests and chi-square tests, respectively, were conducted.

After the descriptive statistics were completed, the correlation among the covariates was examined. If there were any high correlations, those variables were adjusted with one of the related variables. For example, the number of beds was highly correlated with the number of CTs and MRIs; therefore, these two variables were adjusted with the number of beds. This adjustment produced two new variables: the number of CTs per 100 beds and the number of MRIs per 100 beds. These variables were used in the model.

For the main analysis of the factors associated with PHR platform adoption and hospital covariates, a multivariate logistic regression was conducted because the two main outcome variables have a binary scale (having both platforms, Android and iOS, or either single platform). Finally, SAS version 9.4 (SAS Institute Inc., Cary, NC, USA) was used for the data analysis.

\section{Results}

\section{General Characteristics of the Study Subjects}

Table 1 presents the general characteristics of the study hospitals. Among 103 general hospitals, 64 (62.1\%) had adopted both Android and iOS platforms. Thirty-six (35.0\%) and 3 (2.9\%) of the rest of the hospitals had adopted Android only and iOS only, respectively.

Table 2 shows the general features of two groups of hospi- 
tals: those that had adopted both platforms versus those that had adopted only a single platform. A comparison of the two groups showed that the hospitals adopting both platforms were more likely to be tertiary hospitals $(p=0.0047)$ and to have greater numbers of beds $(p<0.0001)$ and CTs. In contrast, hospitals with one platform only had a significantly higher number of MRIs per 100 beds ( $p=0.0428$ ).

The correlation matrix among the independent variables is presented in Table 3. The bottom left-hand side of the table shows figures without adjustment of the number of beds, and the upper right-hand side of the table shows the values adjusted by the number of beds. The high correlation disappeared after the number of beds was adjusted except for the relationship between tertiary hospitals and the number of beds (0.752). This study included this variable, i.e., tertiary

Table 1. Types of PHR platform in hospitals with 100 or more beds

\begin{tabular}{cc}
\hline $\begin{array}{c}\text { Adoption types of PHR } \\
\text { platforms }\end{array}$ & Number of study subjects (\%) \\
\hline All & $103(100)$ \\
Both & $64(62.1)$ \\
Android only & $36(35.0)$ \\
iOS only & $3(2.9)$ \\
\hline
\end{tabular}

status, although there was a high correlation because the role of tertiary hospitals is important and its effect should be controlled.

\section{Analysis of PHR Functionalities}

Table 4 shows an overview of PHR functionalities according to the type of PHR platform focusing on where users can view their booking status, prescription status, and test results or future test schedules. The patterns of functionality of the two platforms were similar to each other. Twenty-one percent (22 cases) of the study hospitals had both platforms having all three functionalities.

Table 5 shows whether the two groups have differences in functionalities. This study found that three functionalities were more frequently observed in the PHR systems of hospitals adopting both platforms compared to those for hospitals adopting only one platform. They were statistically different $(p<0.0001)$.

\section{Factors Associated with Adoption of Both PHR Platforms Table 6 shows the factors associated with the adoption of both PHR platforms compared to the adoption of one plat- form. The numbers of beds (odds ratio $[\mathrm{OR}]=1.004$; confi- dence interval $[\mathrm{CI}], 1.001-1.007 ; p=0.0029)$ and CTs (OR $=6.350 ; \mathrm{CI}, 1.006-40.084 ; p=0.0493)$ were significantly as-}

Table 2. Types of PHR platform in hospitals with 100 or more beds

\begin{tabular}{|c|c|c|c|c|}
\hline \multirow{2}{*}{ Variable } & \multicolumn{3}{|c|}{ Adoption types of PHR platforms } & \multirow{2}{*}{$p$-value } \\
\hline & Both platforms $(n=64)$ & One platform $(n=39)$ & All $(n=103)$ & \\
\hline Foundation (\%) & & & & 0.1367 \\
\hline Private & 75.0 & 87.2 & 79.6 & \\
\hline Public & 25.0 & 12.8 & 20.4 & \\
\hline City location (\%) & & & & 0.0633 \\
\hline Mega-metro city & 67.2 & 48.7 & 60.2 & \\
\hline The others & 32.8 & 51.3 & 39.8 & \\
\hline Tertiary hospital (\%) & & & & 0.0047 \\
\hline Yes & 42.2 & 15.4 & 32.0 & \\
\hline No & 57.8 & 84.6 & 68.0 & \\
\hline Years of operation & $31.0 \pm 17.2$ & $28.0 \pm 12.0$ & $29.8 \pm 15.5$ & 0.3129 \\
\hline Number of beds & $768.0 \pm 468.0$ & $434.9 \pm 266.6$ & $641.9 \pm 427.2$ & $<0.0001$ \\
\hline Number of CTs & $5.7 \pm 3.8$ & $3.2 \pm 1.8$ & $4.8 \pm 3.4$ & $<0.0001$ \\
\hline Number of CTs per 100 beds & $0.79 \pm 0.33$ & $0.74 \pm 0.23$ & $0.77 \pm 0.30$ & 0.3766 \\
\hline Number of MRIs & $3.3 \pm 2.8$ & $1.9 \pm 0.7$ & $2.7 \pm 2.3$ & 0.0002 \\
\hline Number of MRIs per 100 beds & $0.42 \pm 0.16$ & $0.48 \pm 0.16$ & $0.44 \pm 0.17$ & 0.0428 \\
\hline
\end{tabular}

Values are presented as mean \pm standard deviation.

PHR: personal health record, CT: computed tomography, MRI: magnetic resonance imaging. 


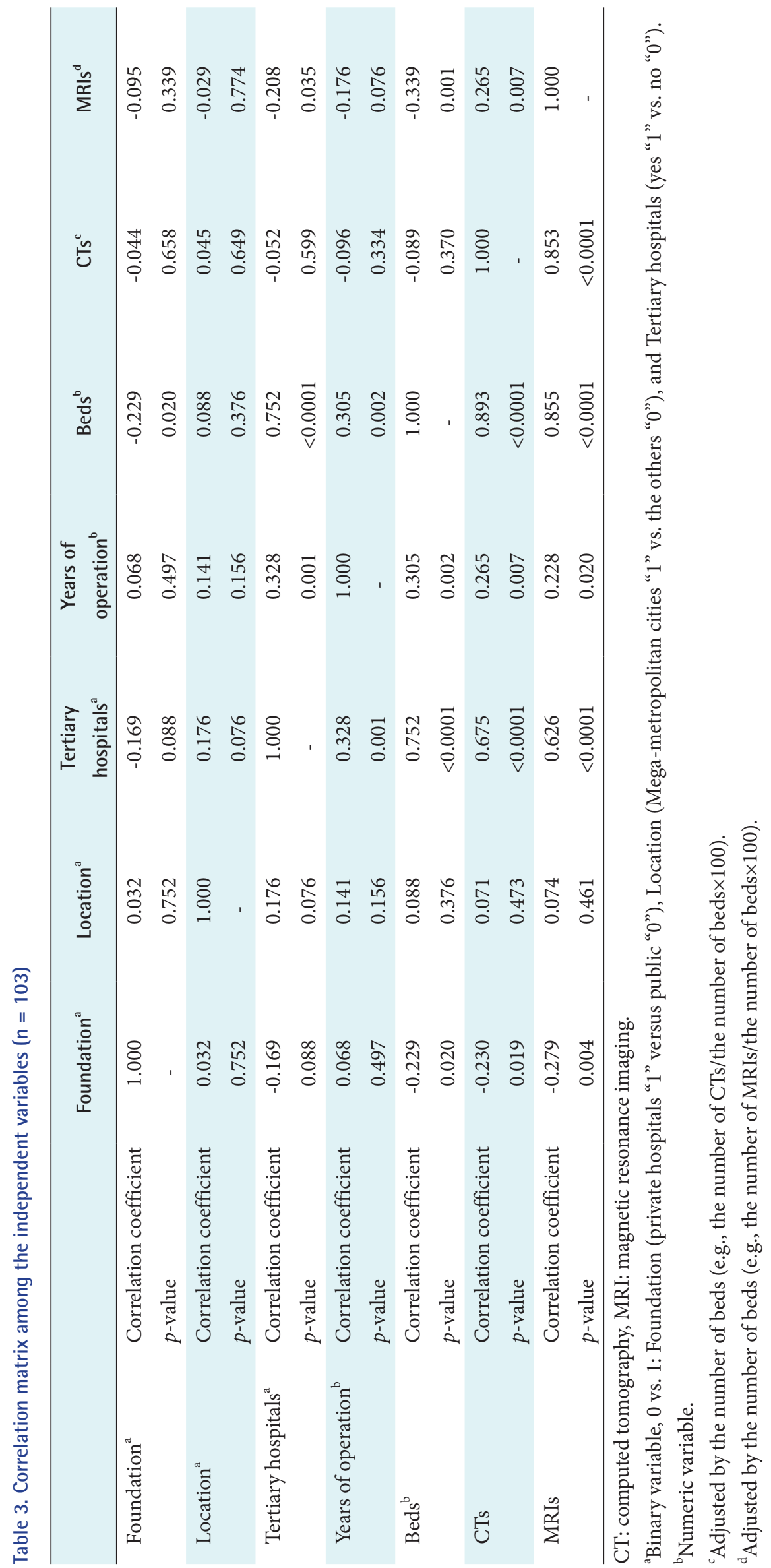




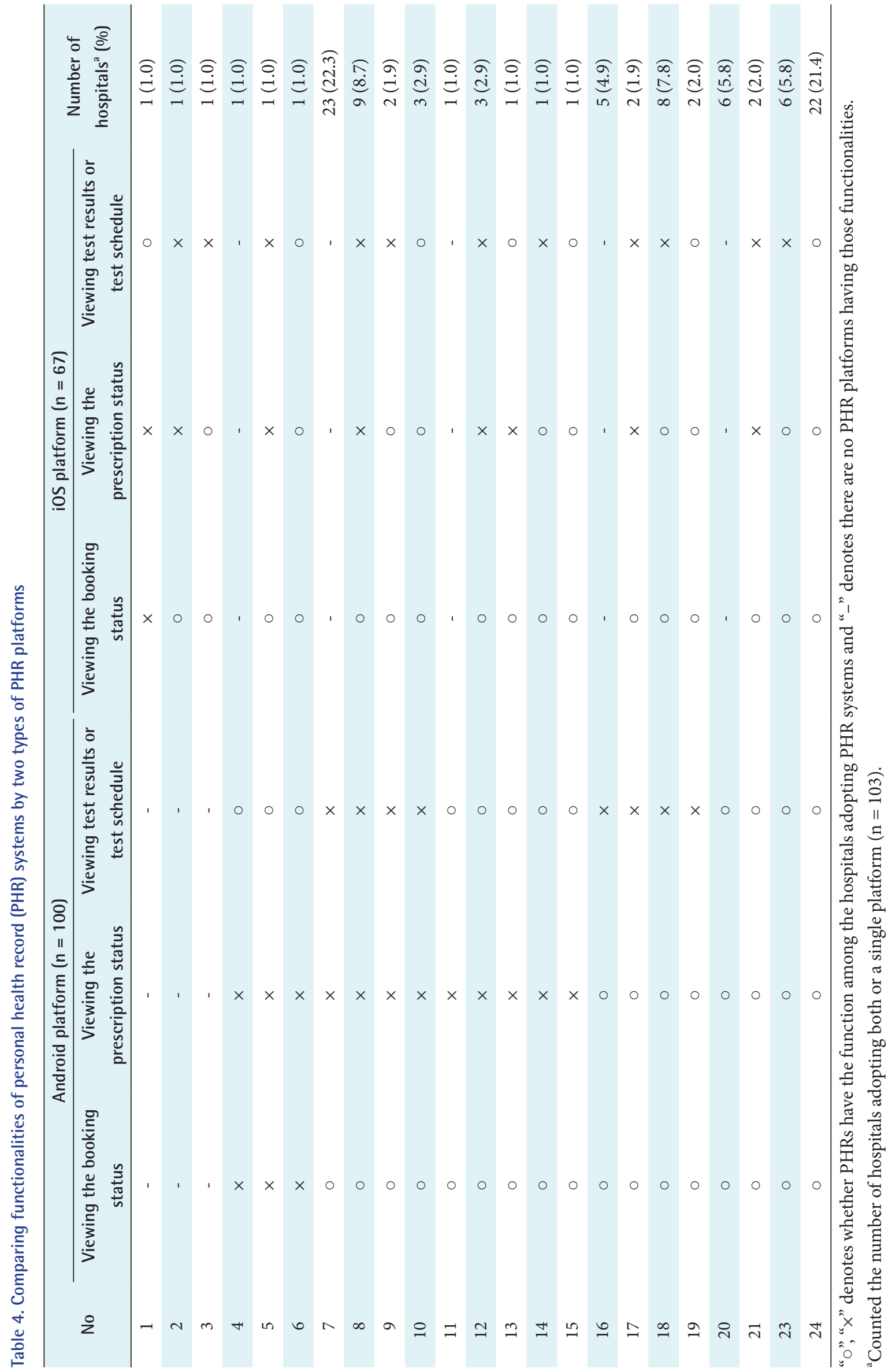


Table 5. PHR functionalities in hospitals with 100 or more beds

\begin{tabular}{|c|c|c|c|c|}
\hline \multirow[b]{2}{*}{ Functionalities } & \multicolumn{3}{|c|}{ Adoption types of PHR platforms } & \multirow[b]{2}{*}{$p$-value } \\
\hline & $\begin{array}{l}\text { Adopting both } \\
\text { platforms }\end{array}$ & $\begin{array}{l}\text { Adopting only one } \\
\text { platform }\end{array}$ & All & \\
\hline Number of study subjects & 64 & 39 & 103 & - \\
\hline Viewing the booking status ${ }^{\mathrm{b}}$ & & & & 0.1411 \\
\hline Yes & 100.0 & 94.9 & 98.1 & \\
\hline No & 0.0 & 5.1 & 1.9 & \\
\hline Viewing the prescription status & & & & $<0.0001$ \\
\hline Yes & 78.1 & 30.8 & 60.2 & \\
\hline No & 21.9 & 69.2 & 39.8 & \\
\hline Viewing test results or test schedule & & & & $<0.0001$ \\
\hline Yes & 67.2 & 23.1 & 50.5 & \\
\hline No & 32.8 & 76.9 & 49.5 & \\
\hline
\end{tabular}

PHR: personal health record.

ancluded three cases with iOS only.

${ }^{\mathrm{b}}$ Fisher exact test result.

Table 6. Factors associated with adoption of both platforms compared to a single platform

\begin{tabular}{lcccc}
\hline \multirow{2}{*}{ Variable } & \multicolumn{3}{c}{ Logistic regression } & \multirow{2}{*}{$p$-value } \\
\cline { 2 - 3 } & OR & Lower Cl & Upper Cl & \\
\hline Private foundation (ref = Public) & 0.706 & 0.200 & 2.492 & 0.5881 \\
Mega-metropolitan city (ref = No) $^{\text {annnn}}$ & 2.370 & 0.894 & 6.281 & 0.0828 \\
Tertiary hospital $^{\text {(ref = No) }}$ & 0.492 & 0.105 & 2.306 & 0.3679 \\
Years of operation & 0.992 & 0.960 & 1.026 & 0.6442 \\
Number of beds & 1.004 & 1.001 & 1.007 & 0.0029 \\
Number of CTs per 100 beds & 6.350 & 1.006 & 40.084 & 0.0493 \\
Number of MRIs per 100 beds & 0.092 & 0.003 & 2.595 & 0.1614 \\
\hline
\end{tabular}

CT: computed tomography, MRI: magnetic resonance imaging, OR: odds ratio, CI: confidence interval.

${ }^{a}$ University hospitals.

sociated with hospitals adopting both platforms.

\section{Discussion}

Many hospitals have adopted PHR systems for their patients. This study investigated the features of hospitals having different PHR platforms and PHR functionalities and targeted two platforms: Android and iOS. This study has three main findings. First, among 103 general hospitals, 64 (62.1\%) hospitals had PHR systems based on both platforms and 39 (37.9\%) hospitals had a single platform, mostly Android. Second, the functions of viewing prescription status and test results or test schedule were frequently observed at hospitals adopting both PHR platforms. Third, the number of beds and CTs was positively associated with the possibility of adopting both platforms compared to adopting only a single platform.

Regarding the types of PHR platforms, this study found that $62.1 \%$ of hospitals had adopted both Android and iOS platforms. Thirty-six percent and $2.9 \%$ had adopted Android only and iOS only, respectively. The dominance of the Android platform might be related to the user market share and development costs. Android and iOS took $74.5 \%$ and $22.9 \%$ market shares at the beginning of 2019, respectively, according to Statcounter reports [25]. Thus, HOs might inevitably have to choose the Android OS to match most users' OS platform. According to the website Salary Expert [26], an iOS developer's annual salary is higher than that of an An- 
droid developer in Korea ( $\$ 48,400$ vs. $\$ 43,200$; currency rate $\$ 1=1,200$ Korean Won).

For the functionalities of PHR platforms, a higher proportion of hospitals having both platforms had systems with three functions compared to those of hospitals only adopting a single platform. The exact reason for the differences was not identified in this study, but a reasonable explanation is that there might have been learned knowledge effects in the process of introducing or developing PHR systems. Knowledge gained from one platform would be easily applied to the second platform, which would affect more detailed functions or the sophistication of PHR systems.

Regarding factors associated with the adoption of both platforms compared to the adoption of a single platform, hospitals adopting both platforms had more hospital beds and a greater number of CTs. Compared to other independent variables, the number of beds and CTs are likely to be factors that lead patients to visit hospitals. Having more beds means that many patients can visit hospitals. Hospitals seek to improve customer service. Patients' mobile phones are a useful and effective tool through which hospitals can easily provide important information to patients and better quality of customer service [27]. PHR use improves patients' interest in and knowledge regarding their health issues [28]. Use of mobile applications could even affect user behaviors [29]. Thus, hospitals having more beds and CTs would be more likely to introduce PHR systems having diverse platforms. This might lead to a high association between two factors. However, it leads to another interesting question of why the number of MRIs was negatively associated with the adoption of both platforms, which warrants further study.

Although this study made several important findings, as noted above, there were some limitations. First, there are many functions in mobile applications of patients regarding PHR systems. This study, however, only considered the three basic functions mentioned in the Methods section: viewing clinic visits, prescription status, and lab results. Second, PHR platforms may be more closely related with a patient's needs. For example, a study showed that patients with one or more chronic diseases were more likely to have higher willingness to adopt PHR than others [30]. However, this study did not consider patients' characteristics, such as the number of patients, their ages, and clinical status, including patients' diagnoses. Future studies should consider these factors. Finally, this study included a variable of whether the hospitals were tertiary although this variable has a high correlation with the number of beds (0.752). The reason we included this variable was that it has a critical role in the market; therefore, we needed to control those effects. To address the issue of a high correlation, we conducted a further analysis with an additional model not having the variable of the hospital's tertiary status. The results of this analysis were nonetheless similar to the current study's results in that the possibility of having both platforms was significantly associated with the number of beds $(p=0.0013)$ and marginally associated with the number of CTs $(p=0.0539)$.

In conclusion, $62.1 \%$ of all general hospitals had adopted both Android and iOS platforms in Korea. Almost 40 percent of general hospitals had adopted a single platform, mostly Android. Hospitals adopting both platforms were more likely to have a function for viewing prescriptions and clinical test results and schedules compared to hospitals adopting a single platform. The possibility of adopting both platforms increases as the numbers of beds and CTs increase. However, there might be some other factors affecting the adoption of different platforms and functions. Patients' characteristics might be one of these factors, and future study could include these variables in the analysis. We hope that the results of this study will contribute to expanding the knowledge base of the healthcare informatics field and other foreign countries regarding the PHR systems of hospitals, especially the platform of PHR systems.

\section{Conflict of Interest}

Young-Taek Park is an editor of Healthcare Informatics Research; however, he did not involve in the peer reviewer selection, evaluation, and decision process of this article. Otherwise, no potential conflict of interest relevant to this article was reported.

\section{Acknowledgments}

We deeply thank Dr. Dong Hwan Kim, MD, Department of Neurosurgery, Pusan National University Hospital and Pusan National University College of Medicine, Korea, for conducting the PHR survey and giving us excellent comments. This study was supported for two years by a Pusan National University Research Grant.

\section{ORCID}

Byung Kwan Choi (http://orcid.org/0000-0001-5244-4592)

Young-Taek Park (http://orcid.org/0000-0002-7574-4165)

Lee-Seung Kwon (http://orcid.org/0000-0003-0143-9384)

Yeon Sook Kim (http://orcid.org/0000-0002-7368-7312) 


\section{References}

1. Lee G, Park JY, Shin SY, Hwang JS, Ryu HJ, Lee JH, et al. Which users should be the focus of mobile personal health records? Analysis of user characteristics influencing usage of a tethered mobile personal health record. Telemed J E Health 2016;22(5):419-28.

2. Jung SY, Kim JW, Hwang H, Lee K, Baek RM, Lee HY, et al. Development of comprehensive personal health records integrating patient-generated health data directly from Samsung S-Health and Apple Health apps: retrospective cross-sectional observational study. JMIR Mhealth Uhealth 2019;7(5):e12691.

3. NHS Digital. Personal health records definition [Internet]. Leeds, UK: NHS Digital; c2020 [cited at 2020 Oct 30]. Available from: https://digital.nhs.uk/services/personal-health-records-adoption-service/personal-healthrecords-adoption-toolkit/initiating-a-personal-healthrecord/personal-health-records-definition.

4. Miller H, Vandenbosch B, Ivanov D, Black P. Determinants of personal health record use: a large population study at Cleveland Clinic. J Healthc Inf Manag 2007;21(3):44-8.

5. Osborn CY, Rosenbloom ST, Stenner SP, Anders S, Muse S, Johnson KB, et al. MyHealthAtVanderbilt: policies and procedures governing patient portal functionality. J Am Med Inform Assoc 2011;18 Suppl 1(Suppl 1):i18-23.

6. Turvey C, Klein D, Fix G, Hogan TP, Woods S, Simon $\mathrm{SR}$, et al. Blue Button use by patients to access and share health record information using the Department of Veterans Affairs' online patient portal. J Am Med Inform Assoc 2014;21(4):657-63.

7. Bouayad L, Ialynytchev A, Padmanabhan B. Patient health record systems scope and functionalities: literature review and future directions. J Med Internet Res 2017;19(11):e388.

8. Graetz I, Huang J, Brand R, Hsu J, Reed ME. Mobile-accessible personal health records increase the frequency and timeliness of PHR use for patients with diabetes. J Am Med Inform Assoc 2019;26(1):50-4.

9. Dameff C, Clay B, Longhurst CA. Personal health records: more promising in the smartphone era? JAMA 2019;321(4):339-40.

10. Google Official Blog. An update on Google Health and Google PowerMeter [Internet]. Mountain View (CA): Google; 2011 [cited at 2020 Oct 30]. Available from: https://googleblog.blogspot.com/2011/06/update-ongoogle-health-and-google.html.
11. Truong K. Microsoft HealthVault is officially shutting down in November [Internet]. New York (NY): MedCity News; 2019 [cited at 2020 Oct 30]. Available from: https://medcitynews.com/2019/04/microsoft-healthvault-is-officially-shutting-down-in-november/.

12. Apple Inc. Apple announces effortless solution bringing health records to iPhone [Internet]. Cupertino (CA): Apple Inc; 2018 [cited at 2020 Oct 30]. Available form: https://www.apple.com/newsroom/2018/01/appleannounces-effortless-solution-bringing-health-recordsto-iPhone/.

13. Comstock J. Apple will launch health records feature at 12 hospitals with iOS 11.3 [Internet]. Portland (ME): MobiHealthNews; 2018 [cited at 2020 Oct 30]. Available from: https://www.mobihealthnews.com/content/ apple-will-launch-health-records-feature-12-hospitalsios-113.

14. Institute for Clinical and Translational Science. Iowa personal health record [Internet]. Iowa City (IA): The University of Iowa; c2020 [cited at 2020 Oct 30]. Available from: https://icts.uiowa.edu/investigators/iowapersonal-health-record.

15. He D, Naveed M, Gunter CA, Nahrstedt K. Security concerns in Android mHealth apps. AMIA Annu Symp Proc 2014;2014:645-54.

16. Sun R, Gregor S, Keating B. Information technology platforms: Conceptualisation and a review of emerging research in IS research. Proceedings of the 26th Australasian Conference on Information Systems (ACIS); 2015 Nov 30-Dec 4; Adelaide, Australia.

17. Thomas LD, Autio E, Gann DM. Architectural leverage: Putting platforms in context. Acad Manag Perspect 2014;28(2):198-219.

18. Scott WR. Organizations: rational, natural, and open systems. 5th ed. Upper Saddle River (NJ): Prentice Hall; 2003.

19. Lee YT, Park YT, Park JS, Yi BK. Association between electronic medical record system adoption and healthcare information technology infrastructure. Healthc Inform Res 2018;24(4):327-34.

20. Menachemi N, Chukmaitov A, Saunders C, Brooks RG. Hospital quality of care: does information technology matter? The relationship between information technology adoption and quality of care. Health Care Manage Rev 2008;33(1):51-9.

21. Ford EW, Hesse BW, Huerta TR. Personal health record use in the united states: forecasting future adoption levels. J Med Internet Res 2016;18(3):e73. 
22. Symons JD, Ashrafian H, Dunscombe R, Darzi A. From EHR to PHR: let's get the record straight. BMJ Open 2019;9(9):e029582.

23. Royal College of Physicians. Personal health record (PHR) landscape review: final report [Internet]. London, UK: Royal College of Physicians; 2016 [cited at 2020 Oct 30]. Available from: https://www.rcplondon. ac.uk/projects/outputs/personal-health-record-phrlandscape-review.

24. Tang PC, Ash JS, Bates DW, Overhage JM, Sands DZ. Personal health records: definitions, benefits, and strategies for overcoming barriers to adoption. J Am Med Inform Assoc 2006;13(2):121-6.

25. Casserly M. iPhone vs Android market share [Internet]. Lanarkshire, UK: Mac Publishing; 2019 [cited at 2020 Oct 30]. Available from: https://www.macworld.co.uk/ feature/iphone-vs-android-market-share-3691861/.

26. Economic Research Institute. Android software developer salary in South Korea [Internet]. Irvine (CA):
Economic Research Institute; 2020 [cited at 2020 Oct 30]. Available from: https://www.erieri.com/salary/job/ android-software-developer/south-korea.

27. Price M, Bellwood P, Kitson N, Davies I, Weber J, Lau F. Conditions potentially sensitive to a personal health record (PHR) intervention, a systematic review. BMC Med Inform Decis Mak 2015;15:32.

28. Park Y, Yoon HJ. Understanding personal health record and facilitating its market. Healthc Inform Res 2020;26(3):248-50.

29. Prapkree L, Sadjadi M, Huffman F, Palacios C. Development and pilot testing of the snackability smartphone application to identify healthy and unhealthy snacks. Healthc Inform Res 2019;25(3):161-72.

30. Determann D, Lambooij MS, Gyrd-Hansen D, de Bekker-Grob EW, Steyerberg EW, Heldoorn M, et al. Personal health records in the Netherlands: potential user preferences quantified by a discrete choice experiment. J Am Med Inform Assoc 2017;24(3):529-36. 